\author{
N. L. S. Martin \\ Department of Physics and Astronomy \\ University of Kentucky \\ Lexington, KY 40506-0055
}

December 1995

PREPARED FOR THE U.S. DEPARTMENT OF ENERGY UNDER GRANT NUMBER DE-FG05-91ER14214

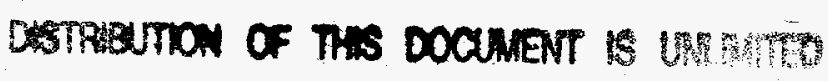

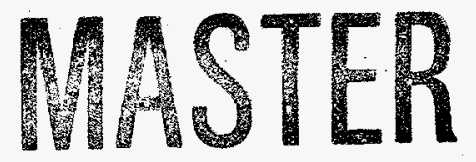




\section{DISCLAIMER}

This report was prepared as an account of work sponsored by an agency of the United States Government. Neither the United States Government nor any agency thereof, nor any of their employees, make any warranty, express or implied, or assumes any legal liability or responsibility for the accuracy, completeness, or usefulness of any information, apparatus, product, or process disclosed, or represents that its use would not infringe privately owned rights. Reference herein to any specific commercial product, process, or service by trade name, trademark, manufacturer, or otherwise does not necessarily constitute or imply its endorsement, recommendation, or favoring by the United States Government or any agency thereof. The views and opinions of authors expressed herein do not necessarily state or reflect those of the United States Government or any agency thereof. 


\section{DISCLAIMER}

Portions of this document may be illegible in electronic image products. Images are produced from the best available original document. 


\title{
Coherent Excitation of Autoionizing Resonances
}

\author{
N.L.S. Martin \\ Department of Physics and Astronomy, University of Kentucky \\ Lexington, Kentucky 40506-0055
}

\section{Introduction}

Experimental investigations are being carried out into interference effects caused by the coherent population, by electron impact, of autoionizing levels and continua of differing total angular momentum and parity. The work provides information on the atomic excitation and ionization mechanisms by charged particle impact.

The experimental technique being used is electron-electron coincidence [or $(e, 2 e)]$ spectrometry. Differing binary $\left(I^{+}\right)$and recoil $\left(I^{-}\right)$lobe intensities in coplanar $(e, 2 e)$ ejected-electron angular distributions may be modeled by by interference cross-terms, between opposite parity final-state continua, that change sign when $\theta_{e j} \rightarrow \theta_{e j}+180^{\circ}$ in the angular distributions. The magnitude of these cross-terms varies rapidly with ejected-electron energy across overlapping autoionizing resonances. The energy variation in the interference terms may be examined by obtaining the sum $\left(I^{+}+I^{-}\right)$and difference $\left(I^{+}-I^{-}\right)$between $(e, 2 e)$ energy spectra measured at ejected-electron angles $180^{\circ}$ apart [1-4].

\section{Experimental Work}

\section{Cadmium}

The coplanar $(e, 2 e)$ spectrometer consists of four principal components: an electron gun, a metal vapor atomic beam oven, a scattered electron spectrometer and an ejected electron spectrometer. The ejected-electron channel incorporates a resistive anode type position sensitive detector. The spectrometers are mounted on two horizontal concentric turntables; the ejected electron is therefore always detected in the scattering plane. The angular range of the scattered spectrometer is $\pm 45^{\circ}$ with respect to the electron beam direction. Because the electron gun is recessed in a side arm, the ejected spectrometer has a useful range of $-30^{\circ} \rightarrow-160^{\circ}$ and $-200^{\circ} \rightarrow-270^{\circ}$, when the scattered spectrometer is at $0^{\circ}$. This range is sufficient to investigate the binary and recoil directions in a single experiment, without recourse to the more usual method of normalizing separate experiments at $\pm \theta_{s c}$. 




Figure 1. (a) Sum and (b) difference $(e, 2 e)$ spectra from $39.2^{\circ}$ magic angle experiment for $\theta_{s c}=12^{\circ}$

A recent major experimental advance has been the development of a novel type of high-voltage trap for the collection of cadmium vapor [5]. The trap makes use of the empirical observation that the deposition of cadmium on surfaces occurs mainly close to high voltage electrodes. This effect seems to have its origins in an attractive force acting on neutral cadmium atoms due to an induced electric-dipole moment in the presence of large electric-field gradients. The trap consists of a central aluminum disc maintained at $+1000 \mathrm{~V}$ with respect to ground potential and mounted at the top of a vertical aluminum cylinder terminated at its bottom end by a triple mesh assembly to provide adequate electrostatic shielding of the interaction region. This device is extremely efficient at the collection of cadmium, resulting in much longer microchannel plate lifetimes.

Measurements are currently being made for a range of scattering angles up to $20^{\circ}$. At each scattering angle pairs of spectra for ejected-electron directions $180^{\circ}$ apart are taken for three angles with respect to the momentum transfer axis: $0^{\circ}$, $39.2^{\circ}$, and $54.7^{\circ}$. These three angles probe different ejected-electron partial waves. At $0^{\circ}$ all partial waves are present, whereas at the other two "magic angles", the $m=0$ components of $\ell=3,2$ are absent respectively since $Y_{\ell 0}\left(\theta_{\mathrm{MAG}}\right)=0$. Because of the angular momentum of ground state $\mathrm{Cd}$ and $\mathrm{Cd}^{+}\left({ }^{1} S_{0}\right.$ and $\left.{ }^{2} S_{\frac{1}{2}}\right)$ each partial wave corresponds to the ionization amplitude of multipolarity $J=\ell$, where $J$ is the 
total angular momentum of the $\mathrm{Cd} 5 s E \ell$ continuum.

Earlier experiments [4] found that at small scattering angles the ionization process is well described by restricting the multipole series to $J=0,1,2$. The measured relative multipole magnitudes (monopole/dipole and quadrupole/dipole) disagreed with PWBA calculated quantities by about a factor of two. The measured relative phases disagreed with the PWBA results by about $\pm \pi / 4$.

At the larger scattering angles of the present experiments, the inclusion of several higher multipoles is required for a description of the ionization process. However, for an intermediate scattering angle where $J=3$ becomes important, but higher terms are still small, the experiments at $39.2^{\circ}$ magic angle experiment "removes" the $J=3$ amplitude and enables a test of the relative $J=0,1,2$ magnitudes and phases. This is shown in Fig 1 for a scattering angle of $12^{\circ}$. In the figure, the theoretical PWBA monopole/dipole and quadrupole/dipole amplitudes have been scaled and phase adjusted by the amounts found from the small angle experiments. It is being investigated whether these corrections are valid for all scattering angles up to $20^{\circ}$.

\section{Helium}

A second $(e, 2 e)$ spectrometer has recently been acquired and is at present being reconfigured. The original spectrometer will continue the experiments in cadmium, while it is intended to devote the new spectrometer to similar types of experiments in helium.

\section{References}

[1] N.L.S. Martin, D.B. Thompson, R.P. Bauman and M. Wilson, "(e,2e) Studies on Cadmium Autoionizing Resonances", J. Phys IV (France), Colloq. 3 C6-69 (1993).

[2] N.L.S. Martin, D.B. Thompson, R.P. Bauman and M. Wilson, "Determination of Complex Ionization Amplitudes by $(e, 2 e)$ Spectroscopy", Phys.Rev.Lett 72, 2163 (1994).

[3] N.L.S. Martin, D.B. Thompson, R.P. Bauman, M. Wilson, J. Jiménez-Mier, C.D. Caldwell and M.O. Krause, "Photoelectron Spectra in Cadmium: Comparison with Theory and $(e, 2 e)$ Spectra", J.Phys.B 27, 3945 (1994).

[4] N.L.S. Martin, D.B. Thompson, R.P. Bauman, M. Wilson, "Investigation of Complex Ionization Amplitudes in Cadmium by $(e, 2 e)$ Spectroscopy", Phys. Rev. A 50, 3878 (1994).

[5] R.P. Bauman and N.L.S. Martin, "An efficient Trap for the Collection of a Cadmium Atomic Beam", Rev. Sci. Instr. 66, 4900 (1995). 